\title{
Valency, ionicity and electronic configuration in rare earths
}

\author{
X. Oudet \\ Laboratoire de Magnétisme, Centre National de la Recherche Scientifique \\ 1, place Aristide-Briand, 92190 Meudon, France
}

\begin{abstract}
Résumé. - L'étude de la correspondance entre les solutions des équations de Dirac et de Schrödinger conduit à retenir pour celles de Schrödinger les valeurs positives et négatives du nombre quantique orbital, ce qui donne l'ensemble des états quantiques et leurs divisions en sous-couches, caractérisées pour les éléments des terres rares par leur division en terres cériques et terres yttriques.
\end{abstract}

\begin{abstract}
The study of the correspondence between the solutions of the equations of Dirac and Schrödinger leads to the necessity of retaining in the case of Schrödinger, positive and negative values for the orbital quantum number, which gives all the quantum states and their division in subshells characterised for the rare earth by their division into light and heavy rare earth.
\end{abstract}

1. Introduction. - The hypothesis of ions such as $\mathrm{Fe}_{\mathrm{III}}^{2+}$ or $\mathrm{Ln}_{\mathrm{III}}^{2+}$ (where the ionicity in arabic numerals is the number of electrons lost or gained by the cation or the anion, and the valency, in roman numeral is the combining or substituting capacity of an element with or by hydrogen) in place of $\mathrm{Fe}^{3+}$, or $\mathrm{Ln}^{3+}$ changes the number of electrons in the unfilled shell and leads us to reconsider the electronic configuration of these shells to interpret their magnetic properties. Good agreement has been obtained with the Dirac theory where the shells $\mathrm{p}, \mathrm{d}$ and $\mathrm{f}$ are divided in subshells $k=l$ or $k=-l-1$, which can be filled successively, especially in the case of rare earth. Verification of this has been obtained by the calculation of the saturation magnetic moment and the Curie constant [1, 2, 3].

The purpose of this work is to show that it is possible to choose the same set of eigenvalues of the orbital angular momentum for Schrödinger's equation as that in Dirac's equation.

2. The division in subshells. - In the periodic table of the elements of Mendeleev the periodicity of the properties is associated with the division of the quantum states in shell $n \mathrm{~s}, n \mathrm{p}, n \mathrm{~d}$ and $n \mathrm{f}$ (if $n$ is the principal quantum number) according to the numbers of states for shell. This result indicates that the cause of the quantum state and the number of those states is independent of the number of electrons in the atom. Hence as our purpose is mainly to compare the number of quantum states in both the Dirac and Schrödinger models we can use a hydrogen like atom.

So let us consider an electron of charge $e$ and mass $m$ at a distance $r$ from a potential $V=-e^{2} Z / r$, where $Z$ is the effective number of protons. In Dirac's model [4] the wave function of a quantum state has four compo- nents each being the product of a spherical harmonic and a radial function $\mathrm{fr}^{-1}$ for two components and $g^{-1}$ for the other two. Let one of the four be $\psi_{3}$ with spherical harmonic $Y_{l}^{m}[5,6]$. In order to study the correspondence between the Dirac and Schrödinger models, it is sufficient to just consider $\psi_{3}$.

Let $k$ be the orbital quantum number ; for a given value of $l$ there are two types of solutions, one with $k=l$ and the other with $k=-l-1$ characteristic of the subshells; then,

$$
\begin{aligned}
& \left(E_{i}+m c^{2}-V\right) f-\hbar c \frac{\mathrm{d} g}{\mathrm{~d} r}-\hbar c \frac{k}{r} g=0 \\
& \left(E_{i}-m c^{2}-V\right) g+\hbar c \frac{\mathrm{d} f}{\mathrm{~d} r}-\hbar c \frac{k}{r} f=0
\end{aligned}
$$

with : $E_{i}=E+m c^{2}$ and $V=-e^{2} Z / r$, and $c$ the light velocity.

According to the value of $k=l$ or $k=-l-1$, $g$ and $f$ are different so that the corresponding states are different and $r \psi_{3}=g_{l} Y_{l}^{m}$ or $g_{-l-1} Y_{l}^{m}$.

For $l=0, k$ only takes the value $k=-1$. For a given value of $k$ there are $2|k|$ possible values of $m$ so that for a given value of $l$ there are $4 l+2$ quantum states. The magnetic quantum number say $u$, that determines the corresponding magnetic moment of the component of the total angular momentum, takes the values $u= \pm\left(\mu-\frac{1}{2}\right)$ with $\mu=m$ for $m \geqslant 1$ and $\mu=|m|+1$ for $m \leqslant 0$. We have $u=j=l+\frac{1}{2}$ for $\mu=|k|=l+1$ and $u=j=l-\frac{1}{2}$ for $\mu=k=l$.

The energy of the system is given by:

$$
\frac{E}{m c^{2}}=\left[1+\frac{\alpha^{2} Z^{2}}{\left[p+\sqrt{k^{2}-\alpha^{2} Z^{2}}\right]^{2}}\right]^{-1 / 2}-1
$$

$\alpha=e^{2} / \hbar c$ is the fine structure constant. 
The elimination of the fine component $f[4,5]$ between equation (1) and (2) gives :

$\frac{\mathrm{d}^{2} g}{\mathrm{~d} r^{2}}+\left[\frac{2 m}{\hbar^{2}}\left(E+\frac{e^{2} Z}{r}\right)-\frac{k(k+1)}{r^{2}}\right] g+\varepsilon g=0$

$\varepsilon g$ is small in comparison with the others, so if one neglects $\varepsilon g$ one has the Schrödinger equation, but with the both possibilities $k=l$ or $k=-l-1$ which conserve the product $k(k+1)$.

This result means that with regard to spin taken as positive orientation we must take into account positive and negative values of the orbital angular momentum. From the point of view of the relative orientations between the observable part of the orbital and spin angular momenta this is equivalent to the Schrödinger model where the orbital angular momentum is always considered as positive and the spin can have positive or negative orientation. But in the reckoning of the quantum states there is for $l>0$ a substantial difference between both the models : in Dirac model for each value of $l$ the orbital angular momentum takes the value $k=l$ and $k=-l-1$ whereas in Schrödinger's present model $k$ is only taken equal to $l$.

Furthermore, when in the Schrödinger model for $l \geqslant 0$, we add to each one of the $2 l+1$ values of $m$, the two values $\pm \frac{1}{2}$ of the spin, we divide the shell into two half-shells with exactly the same number of quantum states. Note that these half-shells do not correspond to the subshells in Dirac model. In fact from the point of view of the relative orientations, with the notation $s=\frac{1}{2}$ for the spin, the quantum states with the total angular momentum $(l-s) \hbar$ have with regard to the spin an orbital angular momentum - $l \hbar$. Thus they belong to the shell preceding the shell $l$, and must have as wave functions the $\psi_{l-1}=y_{l-1} Y_{l-1}^{m}$ where $y_{l-1}$ is the radial function in the Schrödinger model. Hence we must be careful about the inner quantum number $j=l \pm s$. From the above comparison it is seen that in the same shell $l$ the total angular momentum $M$ in each subshell is $M=(k+s) \hbar$ with either $M=(l+s) \hbar$ for $k=l$ or $M=-(l+s) \hbar$ for $k=-l-1$. But the corresponding maximum value of the magnetic quantum number $u$ is $j=l-s$ for $k=l$ and $j=l+s$ for $k=-(l+1)$, as experimentally established by the anomalous Zeeman effect in the alkali metals [7]. So $j$ is the maximum value of $u$ and this can be seen also in the expression of the energy (3) when $k^{2}$ is replaced by $\left(j+\frac{1}{2}\right)^{2}[8]$.

In view of the division in subshells, it is pertinent to bear in mind the division of the rare earths into light rare earths or ceric earths and heavy rare earths or yttric earths based on their chemical and physical properties, which indeed seem to reflect the two $4 \mathrm{f}$ subshells. One of the most striking results in this division is the position of samarium with just six $4 \mathrm{f}$ electrons and its special properties which seem to correspond to the low spin configuration where the first subshell with six quantum states is just filled, a possibility that we have used to interpret the progressive semi conductor metal transition of $\mathrm{SmB}_{6}$ and the phase transformation of SmS [9].

This peculiarity of $4 \mathrm{f}$ electrons comes from the fact that they are deep in the atom core and hence the relativistic correction leading to bifurcation [10] or splitting of the two subshells is quite important. Also the crystal field effects are smaller. However for the d electrons which are outer electrons the crystal field effects dominate and the relativistic correction is very small.

3. The spin-orbit coupling. - As a matter of fact the division in subshells raises the question of the Spin-Orbit coupling since it seems completely defined by the quantum state $k$, which gives the relative orientation of the spin and orbital angular momenta.

To apprehend the problem let us consider $k \hbar$, the orbital angular momentum, and let $\omega_{k}$ be the angular frequency associated with $k$; the corresponding energy is $E_{k}=\frac{1}{2}|k| \hbar \omega_{k}$. Since the total angular momentum is $\left(k+\frac{1}{2}\right) \hbar$, it must have an inner angular frequency $\omega_{\mathrm{s}}$ in such a way that :

$$
E_{k}=\frac{1}{2}|k| \hbar \omega_{k}=\frac{1}{2}\left|k+\frac{1}{2}\right| \hbar \omega_{\mathrm{s}} .
$$

So we have

$$
\omega_{\mathrm{s}}=g \omega_{k} \quad \text { with } \quad g=\frac{k}{k+\frac{1}{2}} ;
$$

and $g$ is nothing other than the splitting factor [11]. It is evident that in the interpretation of the magnetic phenomena $\omega_{s}$ must be introduced in place of $\omega_{k}$ which is done by multiplying the magnetic moment by $g$.

So from this point of view, the $g$ factor, arising from the coupling between orbital and spin angular momenta, appears as a fundamental quantum property characteristic of the subshell.

We think that the electronic configuration according to Dirac's model and the distinction between valency and ionicity will be helpful in understanding metallic rare earth properties as in the case of insulator compounds. 


\section{References}

[1] OUdet, X., 11th Rare Earths Research Conference, Traverse City, Michigan (U.S.A.) 7-10 october (U.S.A.E.C.T.I.C.) 1 (1974) 97.

[2] Oudet, X., Acta. Phys. Pol. A 47 (1975) 789.

[3] OUdET, X., Rare Earths in Modern Science and Technology, 13th Rare Earth Research Conference, Oct. 16-20, 1977, Olgebay Park, W. VA. U.S.A. (Plenum Publishing Corporation) 1978 , p. 453.

[4] Dirac, P. A. M., Proc. R. Soc. (London) A 117 (1928) 610.

[5] Darwin, C. G., Proc. R. Soc. (London) A 118 (1928) 654.
[6] De Broglie, L., L'électron Magnétique (théorie de Dirac) (Hermann, Paris) 1934.

[7] White, H. E., Introduction to Atomic Spectra (Mc Graw-Hill Book Company Inc.) 1934.

[8] Messiah, A., Mécanique quantique (Dunod, Paris) 1960.

[9] OUDET, X., Valence instabilities and Related narrow Band phenomena (Plenum Publishing Corporation) 1977, p. 525.

[10] Тном, R., Modèles mathématiques de la morphogenèse (Union Générale d'Editions, Paris) 1974.

[11] Dirac, P. A. M., Proc. R. Soc. (London) A 118 (1928) 351. 